\title{
A Receptor Tyrosine Kinase Inhibitor, Dovitinib (TKI-258), Enhances BMP-2-Induced Osteoblast Differentiation In Vitro
}

\author{
Yura Lee', Kyoung Jun Bae', Hae Jung Chon', Seong Hwan Kim², Soon Ae Kim ${ }^{3, *}$, and Jiyeon Kim,*
}

\begin{abstract}
Dovitinib (TKI258) is a small molecule multi-kinase inhibitor currently in clinical phase $1 / 1 / / I I I$ development for the treatment of various types of cancers. This drug has a safe and effective pharmacokinetic/pharmacodynamic profile. Although dovitinib can bind several kinases at nanomolar concentrations, there are no reports relating to osteoporosis or osteoblast differentiation. Herein, we investigated the effect of dovitinib on human recombinant bone morphogenetic protein (BMP)-2-induced osteoblast differentiation in a cell culture model. Dovitinib enhanced the BMP-2induced alkaline phosphatase (ALP) induction, which is a representative marker of osteoblast differentiation. Dovitinib also stimulated the translocation of phosphorylated Smad1/5/8 into the nucleus and phosphorylation of mitogen-activated protein kinases, including ERK1/2 and p38. In addition, the mRNA expression of BMP-4, BMP-7, ALP, and OCN increased with dovitinib treatment. Our results suggest that dovitinib has a potent stimulating effect on BMP-2-induced osteoblast differentiation and this existing drug has potential for repositioning in the treatment of bone-related disorders.
\end{abstract}

\section{INTRODUCTION}

In vertebrates, the balance between osteoblastic bone formation and osteoclastic bone resorption controls bone homeostasis. However, decreased osteoblastic activity and increased osteoclastic activity lead to a reduction in bone mineral density and, consequently, increases in the risk of fractures and metabolic skeletal diseases, like osteoporosis (Boyle et al., 2003; Harada and Rodan, 2003). Osteoblasts are the main cells developed from mesenchymal stem cells that contribute to bone

\footnotetext{
${ }^{1}$ Department of Biomedical Laboratory Science, School of Medicine, Eulji University, Daejeon 34824, Korea, ${ }^{2}$ Laboratory of Translational Therapeutics, Korea Research Institute of Chemical Technology, ${ }^{3}$ Department of Pharmacology, School of Medicine, Eulji University, Daejeon 34824, Korea *Correspondence: yeon@eulji.ac.kr (JK); sakim@eulji.ac.kr (SAK)
}

Received 27 October, 2015; revised 16 February, 2016; accepted 25 February, 2016; published online 30 March, 2016

Keywords: ALP, BMP-2, Dovitinib, MAPK, osteoblast differentiation, Smad1/5/8 mineral density and produce markers of osteoblasts, such as alkaline phosphatase (ALP), and bone matrix proteins such as osteocalcin (OCN) and osteopontin (OPN) (Katagiri et al., 1994 Long, 2012). The induction of these markers has been regulated by the degree of cross-talk among mitogen-activated protein kinase (MAPK) pathways such as ERK, JNK, and p38 (Candeliere et al., 2001; Chae et al., 2002; Suzukim et al., 2002; Wu et al., 2006). Among the bone formation stimulating factors, bone morphogenetic proteins (BMPs), which are members of the transforming growth factor- $\beta$ (TGF- $\beta$ ) superfamily, exert stimulating effects on osteoblast differentiation and bone mineralization (Phimphilai et al., 2006). In particular, BMP-2 is a main regulator of osteoblastogenesis and bone regeneration (Rosen, 2009; Wagner et al., 2010).

Many studies have investigated methods for balance recovery between bone formation and resorption. Thus, anabolic agents that stimulate osteoblast differentiation or antiresorptive activity have been developed to attempt to treat bone diseases (Garces and Garcia, 2006; Rosen and Bilezikian, 2001). However, since existing anabolic agents for bone diseases have some limitations in the administration methods and costs, new effective drugs are needed (Garrett, 2007). In recent years, many companies began searching existing drugs to reduce research and development (R\&D) spending and adjust these drugs for other indications via the drug repositioning process (Longman, 2004; Son et al., 2013; Stuart, 2004). This process provides many pharmaceutical companies or investigators an effective way to reduce developmental costs and risks of failure in drug developmental processes such as pharmacokinetic/pharmacodynamic studies and clinical trials.

Dovitinib (TKI258) is a small-molecule multi-kinase inhibitor in phase $1 / 1 / / I I$ clinical trials for the treatment of gastric cancer, pancreatic cancer, advanced breast cancer, multiple myeloma, urothelial cancer, and renal cell carcinoma (ClinicalTrials.gov) (André et al., 2013; Hashinoff et al., 2012). Moreover, dovitinib has a safe and effective pharmacokinetic/pharmacodynamic profile (André et al., 2013; Sarker et al., 2008; Wang et al., 2013). Dovitinib was first designed and synthesized as a multitargeted kinase inhibitor (Trudel et al., 2005). In many studies, dovitinib exerted anticancer activity and antiangiogenic activity through the inhibition of fibroblast growth factor receptor (FGFR) and platelet-derived growth factor receptor (PDGFR) (Lee et al. 2005). This inhibition effect led to an in-depth investigation of dovitinib as a new anticancer drug, but there currently are no reports evaluating bone homeostasis or skeletal diseases. 
Dovitinib Enhances BMP-2-Induced Osteoblast Differentiation

Yura Lee et al.

Table 1. Sequences of the primers used in this study

\begin{tabular}{lll}
\hline Target gene & Forward $\left(5^{\prime}-3^{\prime}\right)$ & Reverse $\left(5^{\prime}-3^{\prime}\right)$ \\
\hline BMP-4 & CCTGGTAACCGAATGCTGAT & AGCCGGTAAAGATCCCTCAT \\
$B M P-7$ & CGATACCACCATCGGGAGTT & AAGGTCTCGTTGTCAAATCG \\
ALP & CACTCAAGGGAGAGGTCCAG & CTGCCCAAGAGAGAAACCTG \\
Osteocalcin & CTCTGTCTCTCTGACCTCAC & GTTTGGCTTAGGGCAGCAC \\
GAPDH & GAGTCAACGGATTTGGTCGT & GATCTCGCTCCTGGAAGATG \\
\hline
\end{tabular}

In this study, we investigated whether dovitinib can regulate the BMP-2-mediated signaling pathway and exert anabolic effects in osteoblast differentiation. Our results indicated that dovitinib has potent stimulating effects in the induction of ALP and mRNA transcription of BMPs and osteogenic markers including ALP and osteocalcin (OCN) through activation of ERK1/2, p38 MAPKs, and phosphorylation of Smad1/5/8. In this study, we evaluated the effect of dovitinib as a potential anabolic agent in osteoblast differentiation of bi-potential mesenchymal precursor $\mathrm{C} 2 \mathrm{C} 12$ cells

\section{MATERIALS AND METHODS}

\section{Materials}

Dovitinib was purchased from Selleck Chemicals (USA) and recombinant human bone morphogenetic protein (BMP)-2 was purchased from R\&D systems, Inc. (USA). All cell culture materials including fetal bovine serum (FBS), DMEM, and antibiotics (100 $\mathrm{U} / \mathrm{ml}$ penicillin and $100 \mu \mathrm{g} / \mathrm{ml}$ streptomycin) were purchased from HyClone (UK). Antibodies against p-ERK1/2, ERK1/2, p-p38, p38, and p-Smad1/5/8 were purchased from Cell Signaling Technology, Inc. (MA, USA). An antibody against actin-horse radish peroxidase (HRP) and Smad1/5/8 were purchased from Santa Cruz Biotechnology, Inc. (USA). The ALP staining kit, MEK inhibitor PD98059, and p38 inhibitor SB202190 were purchased from Sigma Aldrich (USA).

\section{Cell culture and differentiation}

Murine bi-potential mesenchymal precursor C2C12 (mouse myoblast cell line) cells were purchased from ATCC (USA) and maintained in DMEM containing $10 \%$ FBS and $1 \%$ antibiotics in a humidified atmosphere of $5 \% \mathrm{CO}_{2}$ at $37^{\circ} \mathrm{C}$. For osteoblast differentiation, $\mathrm{C} 2 \mathrm{C} 12$ cells were seeded in a 96-well plate for $72 \mathrm{~h}$ and then treated with BMP-2 $(50 \mathrm{ng} / \mathrm{ml})$ in culture media containing $5 \%$ FBS. The medium was changed every 3 days.

\section{Cell viability assay}

The $\mathrm{C} 2 \mathrm{C} 12$ cells $\left(4 \times 10^{3}\right.$ cells/well) were seeded in a 96-well plate for $24 \mathrm{~h}$. Cells were treated with BMP-2 (50 ng/ml) alone or combined with dovitinib in culture media containing 5\% FBS for $72 \mathrm{~h}$. Cell viability was assessed using the Cell Counting Kit (CCK)-8 assay kit (Dojindo Molecular Technologies, Inc., Japan) according to the manufacturer's instructions. The absorbance was measured using the Hidex sense beta plus microplate reader (HIDEX, Finland).

\section{Alkaline phosphatase (ALP) activity assay}

The C2C12 cells $\left(4 \times 10^{3}\right.$ cells/well) were seeded in a 96-well plate and incubated for $24 \mathrm{~h}$. Cells were treated with BMP-2 (50 $\mathrm{ng} / \mathrm{ml}$ ) alone or combined with dovitinib in culture media containing $5 \%$ FBS for $72 \mathrm{~h}$. After incubation, cells were fixed with $3.7 \%$ formaldehyde, rinsed with PBS, and stained with the ALP staining kit according to the manufacturer's instruction. The ALP-positive cells were visualized by phase-contrast light microscopy (Olympus Optical, Japan). The ALP activity was evaluated using the 1-Step ${ }^{T M}$ PNPP Substrate solution (Thermo Fisher scientific, USA) and the absorbance was measured using the Hidex sense beta plus microplate reader (HIDEX, Finland) at $405 \mathrm{~nm}$. Relative ALP activity was normalized by the absorbance of dovitinib only treated group.

\section{Western blot analysis}

After treatment with BMP-2 $(50 \mathrm{ng} / \mathrm{ml})$ alone or combined with dovitinib, cytoplasmic and nuclear fractions of lysates were prepared as described previously (Son et al., 2013). Lysates of cytoplasmic fractions and nuclear fractions were loaded on 8$15 \%$ polyacrylamide gels and transferred to nitrocellulose membranes. Specific primary antibodies were used to detect the expression of proteins. After incubation with HRP-conjugated secondary antibodies, membranes were developed using Signal West Femto Maximum Sensitivity Substrate (Pierce Biotechnology, US) with the LuminoGraph (Atto, USA) chemiluminescent imaging system.

\section{Quantitative real-time PCR ( $q R T-P C R)$ analysis}

The $\mathrm{C} 2 \mathrm{C} 12$ cells were treated with BMP-2 $(50 \mathrm{ng} / \mathrm{ml})$ alone or combined with dovitinib in culture media containing $5 \%$ FBS for $72 \mathrm{~h}$. Total RNA was isolated from $\mathrm{C} 2 \mathrm{C} 12$ cells using TRIzol reagent (Life Technologies, USA), and CDNA was synthesized from $1 \mu \mathrm{g}$ of total RNA using the Enzynomics ${ }^{T M}$ Reverse Transcriptase Kit (Enzynomics, Korea), according to the manufacturer's instructions. Quantitative real-time PCR was performed using the $\mathrm{iQ}^{\text {TM }} \mathrm{SYBR}^{\circledR}$ Green Supermix (Bio-Rad, USA) and the CFX96 ${ }^{\text {TM }}$ Real-Time System (Bio-Rad, USA). The primer sequences used in this study are shown in Table 1. All reactions were run in triplicate, and data were analyzed using the $2^{-\Delta \Delta C}$ method (Livak and Schmittgen, 2001). The internal standard was GAPDH and statistical significance was determined with the Student's $t$-test using GAPDH-normalized $2^{-\Delta \Lambda C}$ Talues (Livak and Schmittgen, 2001).

\section{Statistical analysis}

Data are represented as the mean \pm SEM of at least three independent experiments. Significant differences were evaluated by the Student's $t$-test: ${ }^{\#} P<0.01 ;{ }^{\# \#} P<0.001$ (versus the control); ${ }^{\star} P<0.05$; ${ }^{\star \star} P<0.01$ (versus cells treated with BMP-2 alone); ${ }^{\S} P<0.05 ;{ }^{\S} P<0.01$ (versus cells treated with BMP-2 and dovitinib)

\section{RESULTS}

Dovitinib enhances BMP-2-induced osteoblast differentiation via the Smad1/5/8-mediated signaling pathway in C2C12 cells First, to determine the optimal concentration of dovitinib (Fig. 1A) 
A

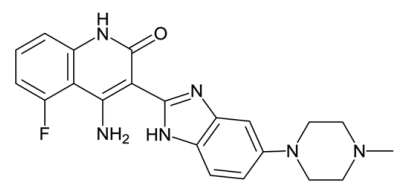

B

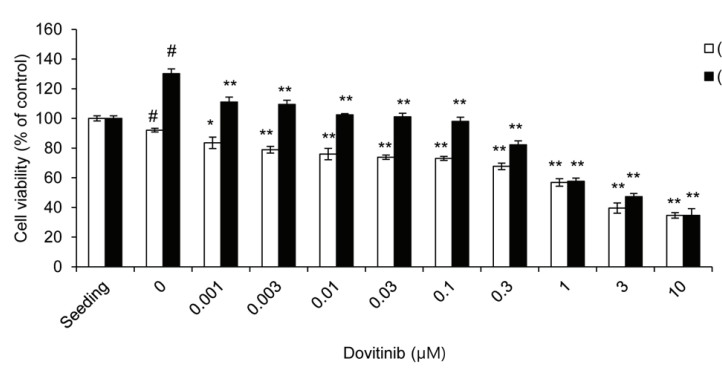

Fig. 1. The effect of dovitinib on the viability of BMP-2-stimultated C2C12 cells. (A) Chemical structure of dovitinib. (B) The $\mathrm{C} 2 \mathrm{C} 12$ cells were treated with BMP-2 $(50 \mathrm{ng} / \mathrm{ml})$ alone or combined with dovitinib for $72 \mathrm{~h}$. Cell viability was evaluated using the CCK-8 assay. Detailed experimental procedures are described in the Materials and Methods. All experiments were performed in triplicate.

for investigating its effect on BMP-2-induced osteoblast differentiation, we assessed its cytotoxicity using a CCK-8 assay. As shown in Fig. 1B, dovitinib exhibited significant cytotoxicity at concentrations of more than $3 \mu \mathrm{M}$, but BMP-2 treated group did not show cytotoxicity at $0.1 \mu \mathrm{M}$. Therefore, non-cytotoxic dosages of dovitinib were used in the following experiments. Next, to evaluate the effect of dovitinib on BMP-2-induced osteoblast differentiation, we examined the expression of ALP, a representative marker for osteoblast differentiation, in $\mathrm{C} 2 \mathrm{C} 12$ cells (Franceschi and lyer, 1992) (Fig. 2A). Treatment with BMP-2 induced the expression of ALP and this was enhanced by dovitinib in a dose-dependent manner. This result was confirmed by an ALP activity assay. As shown in Fig. 2B, BMP-2 stimulated the activity of ALP and dovitinib enhanced BMP-2 induced ALP activity in a dose-dependent manner. Next, to investigate the mechanism of BMP-2-induced osteoblast differentiation, we examined the related signaling pathways. We found BMP-2 activates phosphorylation of $S$ mad1/5/8, which then interacts with Smad4 and subsequently translocates into the nucleus (Cao and Chen, 2005). Dovitinib stimulated the BMP-2mediated accumulation of phosphorylated Smad1/5/8 in a dose-dependent manner (Fig. 2C). However, dovitinib only did not show a stimulating effect on the ALP activity (Supplementary Fig. S1) and phosphorylation of Smad1/5/8 (Supplementary Fig. S2A). These results demonstrated that dovitinib enhances BMP-2-induced osteoblast differentiation through the activation of the Smad1/5/8-mediated signaling pathway.

Dovitinib stimulates the MAPK signaling pathway in BMP-2-induced osteoblast differentiation of $\mathrm{C} 2 \mathrm{C} 12$ cells

Additionally, BMP-2 activates non-Smad signaling such as MAPKs, including ERK1/2, p38, and JNK, in the osteoblast differentiation process (Guicheux et al., 2003; Reilly et al., 2005). Dovitinib enhanced the phosphorylation of ERK $1 / 2$ and p38 (Fig. 3A), but JNK were not changed (data not shown). To confirm the relevance between BMP-2-induced osteoblast differentiation and MAPK activation, we detected the ALP activity
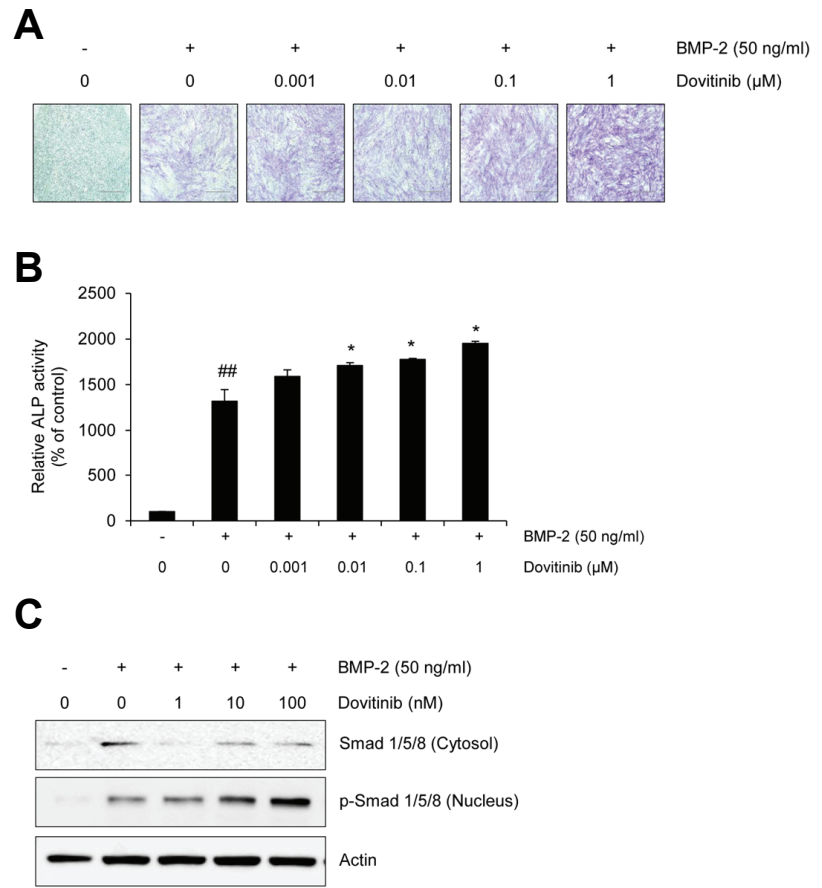

Fig. 2. Dovitinib stimulates the induction of ALP in BMP-2-induced osteoblast differentiation through activation of the Smad1/5/8mediated signaling pathway. (A) The effect of dovitinib on BMP-2induced osteoblast differentiation was detected by visualizing the induction of ALP in C2C12 cells. Scale bars represent $100 \mu \mathrm{m}$. (B) The activity of ALP, when cells were treated with BMP-2 alone or combined with dovitinib, was measured by a microplate reader. All experiments were performed in triplicate. (C) The $\mathrm{C} 2 \mathrm{C} 12$ cells were treated with BMP-2 $(50 \mathrm{ng} / \mathrm{ml})$ alone or combined with dovitinib for 2 days. After 3 days, cells were lysed and fractionated to the cytosol and nuclear portions. The phosphorylation and translocation of Smad1/5/8 into the nucleus were measured by Western blot analysis. Actin was used as a loading control.

in the presence of dovitinib and the MEK inhibitor PD98059 and p38 inhibitor SB202190. The number of ALP-positive cells was increased by dovitinib treatment, but PD98059 and SB202190 suppressed the ALP activity in a dose-dependent manner (Figs. $3 \mathrm{~B}$ and $3 \mathrm{C}$ ). However, dovitinib only did not show dosedependent enhancing effect on the phosphorylation of ERK1/2 and p38 (Supplementary Fig. S2B). These results support that dovitinib exerts a stimulating effect on BMP-2-induced osteoblast differentiation of $\mathrm{C} 2 \mathrm{C} 12$ cells through the activation of MAPK signaling pathways, as well as the Smad-mediated signaling pathway.

\section{Dovitinib activates mRNA expression of osteoblast differentiation marker genes}

During BMP-2-mediated osteoblast differentiation, BMP-2 induces transcription of endogenous BMPs in C2C12 cells. Approximately $20 \mathrm{BMPs}$ of vertebrates originally have osteogenic activity, as well as various other physiological activities (De Biase and Capanna, 2005). Among different BMPs, BMP-2, BMP-4, BMP-5, BMP-6, and BMP-7 are most commonly discovered as osteogenic BMPs. We further investigated whether mRNA expression of these osteogenic BMPs and osteogenic 

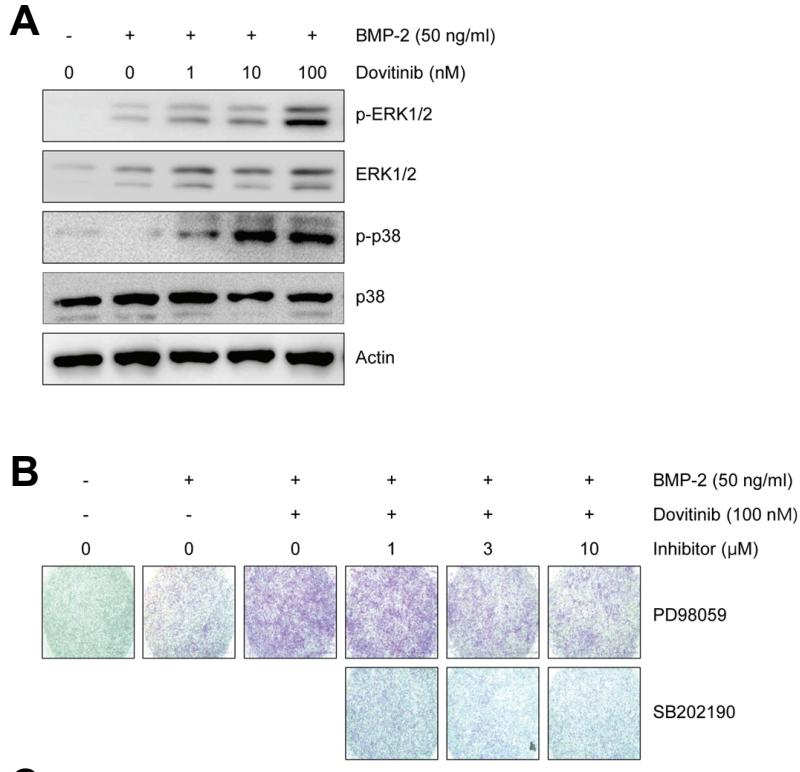

C

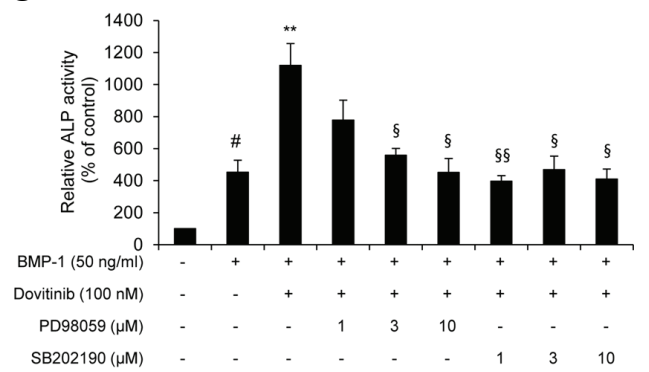

Fig. 3. Dovitinib activates MAPK signaling pathways in BMP-2induced osteoblast differentiation. The $\mathrm{C} 2 \mathrm{C} 12$ cells were treated with BMP-2 $(50 \mathrm{ng} / \mathrm{ml}) \pm$ dovitinib for 10 minutes. (A) The phosphorylation of ERK1/2 and p38 was measured by Western blot analysis. Actin was used as a loading control. (B, C) The C2C12 cells were treated with PD98059 or SB202190 in the presence of BMP-2 $(50 \mathrm{ng} / \mathrm{ml})$ and dovitinib $(100 \mathrm{nM})$ for 3 days. The effect of inhibitors on the BMP-2-induced osteoblast differentiation was detected by the induction of ALP activity in $\mathrm{C} 2 \mathrm{C} 12$ cells. Experiments were performed in triplicate. Scale bars represent $100 \mu \mathrm{m}$.

markers such as ALP, Osteocalcin (OCN), and Runx2 is involved in the osteogenic stimulating effect of dovitinib using real-time PCR analysis. Among osteogenic BMPs, the mRNA expression levels of BMP-4, BMP-7, ALP, and OCN were increased by the combination of BMP-2 and dovitinib in a dosedependent manner (Fig. 4), but the expression levels of other BMPs and Runx2 were not changed (Supplementary Fig. S3). This result indicated that dovitinib enhances the mRNA transcriptional activity of osteogenic markers BMP-4, BMP-7, ALP, and $\mathrm{OCN}$ in the BMP-2-induced osteoblast differentiation process.

\section{DISCUSSION}

In this study, we investigated the osteogenic effect of dovitinib in the BMP-2-mediated osteoblast differentiation of $\mathrm{C} 2 \mathrm{C} 12$ cells. Dovitinib (TKI-258) was originally developed as a multi-targeted receptor tyrosine kinase (RTK) inhibitor and has a potent
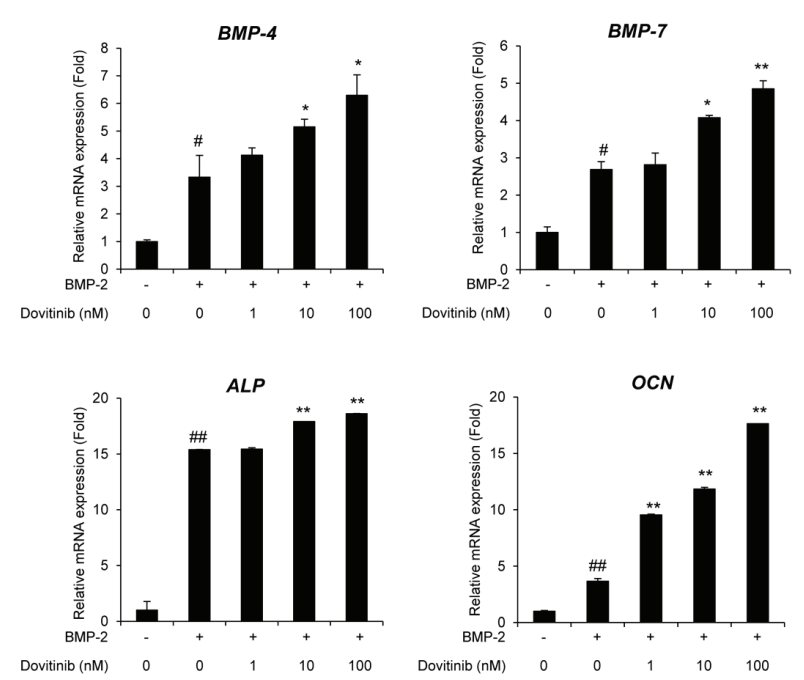

Fig. 4. Dovitinib enhances BMP-2-induced transcriptional activity of osteogenic BMPs. The C2C12 cells were treated with BMP-2 (50 $\mathrm{ng} / \mathrm{ml}$ ) alone or combined with dovitinib in culture media containing $5 \%$ FBS for $72 \mathrm{~h}$. The mRNA expression levels of BMP-4, BMP-7, $A L P$, and osteocalcin were measured by qRT-PCR analysis, with GAPDH used as an internal control. Detailed experimental procedures are described in the "Materials and Methods".

inhibitory effect on the activities of the Class III (FLT3/c-Kit), Class IV (FGFR1/3), and Class V (VEGFR1-4) RTKs (Lopes de Menezes et al., 2005; Porta et al., 2015). In the previous reports, dovitinib has been used as an anti-cancer agent undergoing preclinical or clinical trials (Angevin et al., 2013; Eritja et al., 2014; Kim et al., 2011; Milowsky et al., 2014).

Until now, dovitinib showed anti-cancer activity in many types of human cancers, but there were no specific reports against bone disease. Although dovitinib shows wide ranges of IC50 in many types of cancer cells, this study demonstrated that relatively low concentrations of dovitinib could exert a stimulating effect on the BMP-2-induced osteoblast differentiation of C2C12 cells without significant cytotoxicity. As mentioned in previous reports, dovitinib showed anti-cancer activity through inhibition of FGFRs, PDGFRs, and VEGFRs (Angevin et al., 2013; Eritja et al., 2014; Kim et al., 2011; Milowsky et al., 2014). However, in $\mathrm{C} 2 \mathrm{C} 12$ cells, BMP-2 increased the proliferation of C2C12 cells and dovitinib did not exert significant cytotoxicity. This result implies that relatively low concentrations of dovitinib could not inhibit the activity of FGFRs or PDGFRs to affect the cell viability of BMP-2-treated $\mathrm{C} 2 \mathrm{C} 12$ cells. Although, osteoblast differentiation can be mediated by FGF/FGFR or PDGF/PDGFR signaling, multiple signaling networks including TGF- $\beta / B M P$, MAPK, Smad, Akt/mTOR, and Wnt signaling and transcription factors tightly regulate osteogenesis or bone formation (Caverzasio et al., 2013; Guicheux et al., 2003; Hipskind and Bilbe, 1998; Kobayashi et al., 2015; Marie et al., 2012; Rahman et al., 2015). Additionally, among the FGFs, FGF-8 suppresses BMP2-induced osteoblast differentiation through the inhibition of ERK pathway (Katsuyama et al., 2015). This report also support our results that dovitinib might act as a FGF-8 inhibitor to activate BMP-2-induced osteoblast differentiation. In this regard we suggest that dovitinib showed synergistic effect on osteoblast differentiation process of $\mathrm{C} 2 \mathrm{C} 12$ cells via BMP-2-induced 
MAPK and Smad signaling cascades.

The biopharmaceutical industry has rapidly grown and invested a lot of money for the R\&D of new drugs; however, the number of approved new drugs has not kept pace with the increase in $R \& D$ spending. To reduce the $R \& D$ expenditures and increase the success rate, many companies apply various strategies in drug development. Of these cost-reducing approaches, drug repositioning is a new method to search existing developed drugs (Ashburn and Thor, 2004; Chong and Sullivan, 2007). Drug repositioning allows companies to develop new drugs through changing the scope of the original medical indication, leading to faster processing, while reducing the risks of failure in drug discovery and development. In a previous report, we investigated the potential of the anti-cancer drug CX4945 as a regulator of osteoblast and osteoclast differentiation (Son et al., 2013). This report provided a possibility that anticancer drugs in clinical trials or FDA approved can be used for other indications.

Based on the concept of drug repositioning, we also evaluated the effect of dovitinib on BMP-2-induced osteoblast differentiation. Recent reports provide new information about therapeutic applications of anti-cancer agents for the treatment of another disease or synergistic effects with exiting drugs (Beeharry et al., 2014; Bharadwaj et al., 2015; Hanusova et al., 2015; Pemovska et al., 2015; Song et al., 2015). Published reports mainly represent that dovitinib exerts anti-cancer activity through inhibition of FGFRs, PDGFRs, and VEGFRs in various types of cancers (Angevin et al., 2013; Eritja et al., 2014; Kim et al., 2011; Milowsky et al., 2014). Targeted therapies, associated with specific types of diseases, have improved in drug discovery and development process. However, increasing resistance rates of tumor cells against targeted chemotherapies induce fail in the development of new drugs. Compared with the previous reports, our study did not show dovitinib induced cytotoxicity and inhibitory activity on the expression of FGFRs or PDGFRs in $\mathrm{C} 2 \mathrm{C} 12$ cells. Although the biochemical activity of dovitinib was unpredictable and not consistent with published results, our results represent a novel effect of dovitinib in BMP-2mediated signaling. Our key findings indicated that BMP-2 induces osteogenic metabolisms in $\mathrm{C} 2 \mathrm{C} 12$ cells through activation of Smad1/5/8 and MAPK mediated signaling and the transcriptional activity of osteogenic BMPs, such as BMP-4 and BMP-7. This process of BMP-2-induced osteoblast differentiation was enhanced by combined treatment with dovitinib, without a significant cytotoxic effect.

Bone homeostasis is regulated by the ongoing balance between osteoclastic bone resorption and osteoblastic bone formation (Harada and Rodan, 2003). The physiological imbalance in bone remodeling between the differentiation of osteoblasts and osteoclasts results in the bone mass decrease and skeletal disorders such as osteoporosis (Goltzman, 2002). While most of approved drugs regulating bone metabolism mainly suppresses bone resorption through the inhibition of osteoclast differentiation, development of anabolic drugs that induces osteoblast differentiation is not sufficient yet (Goltzman, 2002). In this regard, our study provides a potent opportunity that tyrosine kinase inhibitors can be used for the treatment of osteoporosis. But in fact, we did not evaluate another effect of dovitinib yet whether it has enhancing activity on in vivo bone formation (or mineralization) and inhibitory activity on the osteoclast differentiation. Since bone homeostasis is regulated by osteoblast differentiation and osteoclast differentiation, we thought that further investigations for the identification of dovitinib activity for bone homeostasis will be needed in future.
Here, we showed the potential for an existing drug through repositioning. This could help reduce the time to discover a novel drug candidate and the optimization of pharmacological characteristics. This study utilized an efficient method for the development of new drugs that has a cost-saving effect on future $R \& D$ processes.

Note: Supplementary information is available on the Molecules and Cells website (www.molcells.org).

\section{ACKNOWLEDGMENTS}

This research was supported by Eulji University in 2014 and the Basic Science Research Program through the National Research Foundation (NRF) of Korea, funded by the Ministry of Science, ICT \& Future Planning (NRF-2014R1A1A1002349).

\section{REFERENCES}

André, F., Bachelot, T., Campone, M., Dalenc, F., Perez-Garcia, J.M., Hurvitz, S.A., Turner, N., Rugo, H., Smith, J.W., Deudon, S. et al. (2013). Targeting FGFR with dovitinib (TKI258): preclinical and clinical data in breast cancer, Clin. Cancer Res. 19, 36933702.

Angevin, E., Lopez-Martin, J.A., Lin, C.C., Gschwend, J.E., Harzstark, A., Castellano, D., Soria, J.C., Sen, P., Chang, J., Shi, M., al. (2013). Phase I study of dovitinib (TKI258), an oral FGFR, VEGFR, and PDGFR inhibitor, in advanced or metastatic renal cell carcinoma. Clin. Cancer Res. 19, 1257-1268.

Ashburn ,T.T., and Thor, K.B. (2004). Drug repositioning: identifying and developing new uses for existing drugs. Nat. Rev. Drug Discov. 3, 673-683.

Beeharry, N., Banina, E., Hittle, J., Skobeleva, N., Khazak, V., Deacon, S., Andrake, M., Egleston, B.L., Peterson, J.R., Astsaturov, I., et al. (2014). Re-purposing clinical kinase inhibitors to enhance chemosensitivity by overriding checkpoints. Cell Cycle 13, 21722191.

Bharadwaj, U., Eckols, T.K., Kolosov, M., Kasembeli, M.M., Adam, A., Torres, D., Zhang, X., Dobrolecki, L.E., Wei, W., Lewis, M.T., et al. (2015). Drug-repositioning screening identified piperlongumine as a direct STAT3 inhibitor with potent activity against cancer. Oncogene 34, 1341-1353.

Boyle, W.J., Simonet, W.S., and Lacey, D.L. (2003). Osteoclast differentiation and activation, Nature 423, 337-342.

Candeliere, G.A., Liu, F., and Aubin, J.E. (2001). Individual osteoblasts in the developing calvaria express different gene repertoires, Bone 28, 351-361.

Cao, X., and Chen, D. (2005). The BMP signaling and in vivo bone formation. Gene. 357, 1-8.

Caverzasio, J., Biver, E., and Thouverey, C. (2013). Predominant role of PDGF receptor transactivation in Wnt3a-induced osteoblastic cell proliferation. J. Bone Miner. Res. 28, 260-270.

Chae, H.J., Jeong, B.J., Ha, M.S., Lee, J.K., Byun, J.O., Jung, W.Y., Yun, Y.G., Lee, D.G., Oh, S.H., and Chae, S.W., et al. (2002). ERK MAP kinase is required in 1, 25(OH)2D3-induced differentiation in human osteoblasts, Immunopharmacol. Immunotoxicol. 24 31-41.

Chong, C.R., and Sullivan, D.J. Jr. (2007). New uses for old drugs. Nature 448, 645-646.

De Biase, P., and Capanna, R. (2005). Clinical applications of BMPs. Injury 36, S43-46.

Eritja, N., Domingo, M., Dosil, M.A., Mirantes, C., Santacana, M., Valls, J., Llombart-Cussac, A., Matias-Guiu, X., and Dolcet, X. (2014). Combinatorial therapy using dovitinib and ICl182.780 (fulvestrant) blocks tumoral activity of endometrial cancer cells. Mol. Cancer Ther. 13, 776-787.

Franceschi, R.T., and lyer, B.S. (1992). Relationship between collagen synthesis and expression of the osteoblast phenotype in MC3T3-E1 cells. J. Bone Miner. Res. 7, 235-246.

Garces, C., and Garcia, L.E. (2006). Combination of anabolic and antiresorptive agents for the treatment of osteoporosis, Maturitas 54, 47-54.

Garrett, I.R. (2007). Anabolic agents and the bone morphogenetic 
protein pathway, Curr. Top. Dev. Biol. 78, 127-171.

Goltzman, D. (2002). Discoveries, drugs and skeletal disorders. Nat. Rev. Drug Discov. 1, 784-796.

Guicheux, J., Lemonnier, J., Ghayor, C., Suzuki, A., Palmer, G., Caverzasio, J. (2003). Activation of p38 mitogen-activated protein kinase and c-Jun-NH2-terminal kinase by BMP-2 and their implication in the stimulation of osteoblastic cell differentiation. J. Bone Miner. Res. 18, 2060-2068.

Hanusova, V., Skalova, L., Kralova, V., and Matouskova, P. (2015). Potential anti-cancer drugs commonly used for other indications. Curr. Cancer Drug Targets 15, 35-52.

Harada, S. and Rodan, G.A. (2003). Control of osteoblast function and regulation of bone mass. Nature 423, 349-355.

Hasinoff, B.B., Wu, X., Nitiss, J.L., Kanagasabai, R., and Yalowich, J.C. (2012). The anticancer multi-kinase inhibitor dovitinib also targets topoisomerase I and topoisomerase II, Biochem. Pharmacol. 84, 1617-1626.

Hipskind, R.A. and Bilbe, G. (1998). MAP kinase signaling cascand gene expression in osteobldsts. Front Biose. 3, d804-816. s

Katagiri, T., Yamaguchi A., Komaki, M., Abe, E., Takahashi, N., Ikeda, T., Rosen, V., Wozney, J.M., Fujisawa-Sehara, A., and Suda, T. (1994). Bone morphogenetic protein-2 converts the differentiation pathway of $\mathrm{C} 2 \mathrm{C} 12$ myoblasts into the osteoblast lineage, J. Cell Biol. 127, 1755-1766.

Katsuyama, T., Otsuka, F., Terasaka, T., Inagaki, K., TakanoNarazaki, M., Matsumoto, Y., Sada, K.E., and Makino, H. (2015). Regulatory effects of fibroblast growth factor-8 and tumor necrosis factor- $\alpha$ on osteoblast marker expression induced by bone morphogenetic protein-2. Peptides 73, 88-94.

Kim, K.B., Chesney, J., Robinson, D., Gardner, H., Shi, M.M., and Kirkwood, J.M. (2011). Phase I/II and pharmacodynamic study of dovitinib (TKI258), an inhibitor of fibroblast growth factor receptors and VEGF receptors, in patients with advanced melanoma. Clin. Cancer Res. 17, 7451-7461.

Kobayashi, Y., Uehara, S., Nobuyuki, U., and Takahashi, N. (2015). Regulation of bone metabolism by Wnt signals. J. Biochem. pii: mvv124.

Lee, S.H., Lopes de Menezes, D., Vora, J. Harris, A., Ye, H., Nordahl, L., Garrett, E., Samara, E., Aukerman, S.L., Gelb, A.B., and Heise, C. (2005). In vivo target modulation and biological activity of CHIR-258, a multitargeted growth factor receptor kinase inhibitor, in colon cancer models, Clin. Cancer Res. 11, 3633-3641.

Livak, K.J., and Schmittgen, T.D. (2001). Analysis of relative gene expression data using real-time quantitative PCR and the 2(Delta Delta C(T)) Method. Methods 25, 402-408.

Long, F. (2012). Building strong bones: molecular regulation of the osteoblast lineage, Nat. Rev. Mol. Cell Biol. 13, 27-38.

Longman, R. (2004). Pharmaceutical strategies: jumpstart to products, In Vivo 22, 17

Lopes de Menezes, D.E., Peng, J., Garrett, E.N., Louie, S.G., Lee, S.H. Wiesmann, M., Tang, Y. Shephard, L., Goldbeck, C., Oei, et al. (2005). CHIR-258: a potent inhibitor of FLT3 kinase in experimental tumor xenograft models of human acute myelogenous leukemia. Clin. Cancer Res. 11, 5281-5291.

Marie, P.J., Miraoui, H., and Sévère, N. (2012). FGF/FGFR signaling in bone formation: progress and perspectives. Growth Factors 30, 117-123.

Milowsky, M.I., Dittrich, C., Durán, I., Jagdev, S., Millard, F.E., Sweeney, C.J., Bajorin, D., Cerbone, L., Quinn, D.I., Stadler, et al. (2014). Phase 2 trial of dovitinib in patients with progressive FGFR3-mutated or FGFR3 wild-type advanced urothelial carcinoma. Eur. J. Cancer 50, 3145-3152.

Pemovska, T., Johnson, E., Kontro, M., Repasky, G.A., Chen, J., Wells, P., Cronin, C.N., McTigue, M., Kallioniemi, O., Porkka, K., et al. (2015). Axitinib effectively inhibits BCR-ABL1(T315I) with a distinct binding conformation. Nature 519, 102-105.

Phimphilai, M., Zhao, Z., Boules, H., Roca, H., and Franceschi, R.T. (2006). BMP signaling is required for RUNX2-dependent induction of the osteoblst phenotype, J. Bone Miner. Res. 21, 637-646.

Porta, C., Giglione, P., Liguigli, W., and Paglino, C. (2015). Dovitinib (CHIR258, TKI258): structure, development and preclinical and clinical activity. Future Oncol. 11, 39-50.

Rahman, M.S., Akhtar, N., Jamil, H.M., Banik, R.S., and Asaduzzaman, S.M. (2015). TGF- $/$ /BMP signaling and other molecular events: regulation of osteoblastogenesis and bone formation. Bone Res. 3, 15005.

Reilly, G.C., Golden, E.B., Grasso-Knight, G., and Leboy, P.S. (2005). Differential effects of ERK and p38 signaling in BMP-2 stimulated hypertrophy of cultured chick sternal chondrocytes. Cell Commun. Signal. 3, 3 .

Rosen, V. (2009). BMP2 signaling in bone development and repair, Cytokine Growth Factor Rev. 20, 475-480.

Rosen, C.J., and Bilezikian, J.P. (2001). Clinical review 123: anabolic therapy for osteoporosis, J. Clin. Endocrinol. Metab. 86, 957-964.

Sarker, D., Molife, R., Evans, T.R., Hardie, M., Marriott, C., Butzberger-Zimmerli, P., Morrison, R., Fox, J.A., Heise, C., Louie, S., et al. (2008). A phase I pharmacokinetic and pharmacodynamic study of TKI258, an oral, multitargeted receptor tyrosine kinase inhibitor in patients with advanced solid tumors, Clin. Cancer Res. 14, 2075-2081.

Son, Y.H., Moon, S.H., and Kim, J. (2013). The protein kinase 2 inhibitor CX-4945 regulates osteoclast and osteoblast differentiation in vitro, Mol. Cells 36, 417-423

Song, M., Kim, S.H., and Yoon, S.K. (2015). Cabozantinib for the treatment of non-small cell lung cancer with KIF5B-RET fusion. An example of swift repositioning. Arch. Pharm. Res. 38, 21202123.

Stuart, M. (2004). Rediscovering existing drugs, Start-Up 9, 23-30.

Suzukim, A., Guicheux, J., Palmer, G., Miura, Y., Oiso, Y., Bonjour J., and Caverzasio, J.P. (2002). Evidence for a role of p38 MAP kinase in expression of alkaline phosphatase during osteoblastic cell differentiation, Bone 30, 91-98.

Trudel, S., Li, Z.H., Wei, E., Wiesmann, M., Chang, H., Chen, C., Reece, D., Heise, C., and Stewart, A.K. (2005). CHIR-258, a novel, multitargeted tyrosine kinase inhibitor for the potential treatment of $\mathrm{t}(4 ; 14)$ multiple myeloma, Blood 105, 2941-2948.

Wagner, D.O., Sieber, C., Bhushan, R., Borgermann, J.H., Graf, D., and Knaus, P. (2010). BMPs: from bone to body morphogenetic proteins, Sci. Signal 3, mr1.

Wang, X., Kay, A., Anak, O., Angevin, E., Escudier, B., Zhou, W. Feng, Y., Dugan, H., and Schran, M. (2013). Population pharmacokinetic/pharmacodynamic modeling to assist dosing schedule selection for dovitinib, J. Clin. Pharmacol. 53, 14-20.

Wu, C.C., Li, Y.S., Haga, J.H., Wang, N., Lian, I.Y., Su, F.C., Usamim, S., and Chien, S. (2006). Roles of MAP kinases in the regulation of bone matrix gene expressions in human osteoblasts by oscillatory fluid flow, J. Cell. Biochem. 98, 632-641. 